\title{
Comprometimento Executivo nas Fases Leve à Grave da Doença de Parkinson
}

\author{
Greici Rössler Macuglia \\ Universidade Federal do Rio Grande do Sul \\ Porto Alegre, RS, Brasil \\ Carlos Roberto de Mello Rieder \\ Universidade Federal de Ciências da Saúde de Porto Alegre \\ Porto Alegre, RS, Brasil \\ Lais Broch Trentini \\ Universidade Federal do Rio Grande do Sul \\ Porto Alegre, RS, Brasil \\ Nelson Hauck Filho \\ Universidade São Francisco \\ Itatiba, SP, Brasil \\ André Luiz Moraes \\ Rosa Maria Martins de Almeida \\ Universidade Federal do Rio Grande do Sul \\ Porto Alegre, RS, Brasil

\section{RESUMO}

\begin{abstract}
A Doença de Parkinson (DP) é uma desordem neurodegenerativa caracterizada por disfunções motoras, mas também marcada por alterações executivas. O presente estudo investigou funções executivas em indivíduos saudáveis e pacientes parkinsonianos com grau variável de comprometimento. Foram avaliados 30 controles saudáveis e 40 pacientes com DP utilizando os instrumentos Hoehn e Yahr, WAIS-III, MEEM, MoCA, Stroop e BADS. Pacientes com DP evidenciaram significativo prejuízo nas funções executivas, quando comparados às pessoas do grupo controle. Houve um percentual de $72,5 \%$ de pacientes com disfunção executiva (DE), distribuídos em todos os estágios da DP, sendo que mulheres, indivíduos com menor escolaridade e aqueles com idade avançada apresentaram disfunções mais severas do que os demais pacientes. Portanto, o número de pacientes com alterações executivas foi significativo, não estando estas relacionadas ao agravamento da doença. No entanto, algumas variáveis dos indivíduos da amostra se mostraram protetivas para o agravamento das disfunções.
\end{abstract}

Palavras-chave: Doença de Parkinson; Avaliação neuropsicológica; Funções executivas.

\section{ABSTRACT}

Executive Impairment in Mild to Severe Stages of Parkinson's Disease

Parkinson's disease (PD) is a neurodegenerative disorder characterized by motor dysfunction, but also by remarkable executive changes. In the present study, we sought to investigate executive functions in healthy controls and parkinsonian patients with varying degrees of impairment. We evaluated 30 healthy controls and 40 PD patients using the instruments Hoehn and Yahr, UPDRS, WAIS-III, MMSE, MoCA, Stroop and BADS. PD patients showed significant impairment in executive functions when compared to people of the control group. There was a percentage of $72.5 \%$ of patients with executive dysfunction (ED), distributed in all stages of PD, and women, individuals with less education and those with advanced age showed more severe disorders than other patients. Therefore, the number of patients with executive changes was significant, not being those related to the worsening of the disease. However, some variables of the sample proved protective for worsening dysfunction.

Keywords: Parkinson's Disease; Neuropsychology assessment; Executive functions.

\section{RESUMEN}

\section{Deterioro Ejecutivo en Fase Leve a Severa de la Enfermedad De Parkinson}

Enfermedad de Parkinson (EP) es un trastorno neurodegenerativo caracterizado por la disfunción motora, pero también marcado por cambios ejecutivos. El presente estudio investigó funciones ejecutivas en individuos saludables controles y pacientes con EP con variados grados de severidad. Un total de 30 controles sanos y 40 pacientes con EP fueron evaluados utilizándose los instrumentos Hoehn y Yahr, UPDRS, WAIS-III, MMSE, MoCA, Stroop y BADS. Pacientes con EP mostraron un deterioro significativo de las funciones ejecutivas en comparación con aquellos en el grupo control. Hubo un porcentaje de $72.5 \%$ de los pacientes con disfunción ejecutiva (ED), distribuidos en todas las etapas de la EP, y las mujeres, las personas con menos educación y aquellos con edad avanzada mostraron trastornos más graves que otros pacientes. Por lo tanto, el número de pacientes con cambios ejecutivos fue significativa, no siendo las relacionadas con el empeoramiento de la enfermedad. Sin embargo, algunas variables de la muestra resultó protectora por empeoramiento de la disfunción.

Palabras clave: Enfermedad de Parkinson; Evaluación neuropsicológica; Funciones ejecutivas. 
A doença de Parkinson (DP) caracteriza-se fundamentalmente pela ocorrência de sintomatologia motora, embora alterações cognitivas estejam também intimamente relacionadas à patologia (Kamei, Morita, Serizawa, Mizutani, \& Hirayanagi, 2010). De fato, essas alterações tendem a se mostrar tão incapacitantes quanto os sinais motores (Campos-Sousa, CamposSousa, Ataíde, Soares, \& Almeida, 2010). Dentre os prejuízos cognitivos mais comuns na DP, estão as alterações das funções executivas (FE), que estão presentes, em muitos casos, desde os primeiros estágios da doença, e tendem a se agravar, podendo resultar em demência (Mckinlay, Grace, Dalrymple-Alford, \& Roger, 2010).

As FE atingiram seu ápice na espécie humana e resultam diretamente da atividade desempenhada pelo córtex pré-frontal (CPF), além de outras áreas corticais e subcorticais do cérebro relacionadas aquele (Galhardo, Amaral, \& Vieira, 2009). As FE são um conjunto abrangente de habilidades que permitem que um indivíduo direcione seu funcionamento cognitivo, comportamental e emocional a metas, relacionando-se com a habilidade de responder de forma adaptativa a novas situações. Dessa forma, para que um indivíduo possa atingir determinado objetivo, necessita formular um plano de ação, integrar informações de experiências passadas e presentes, fazer um planejamento, monitorar as consequências desse plano e alterá-lo, caso as consequências não sejam adequadas (Hamdan \& Pereira, 2009). Nesse sentido, diversos processos cognitivos compõem as $\mathrm{FE}$, tais como planejamento, tomada de decisões, memória operacional (memória de trabalho), sequenciação, controle inibitório, flexibilidade cognitiva, categorização, resolução de problemas, raciocínio verbal, atenção e fluência (Chan, Shun, Toulopoulou, \& Chen, 2008; Malloy-Diniz, Paula, Loschiavo-Alvares, Fuentes, \& Leite, 2010;).

As disfunções executivas (DE), isto é, o comprometimento das funções executivas, estão relacionadas a muitas das manifestações cognitivas comprometidas em pacientes com DP, principalmente em pacientes que apresentam maior idade e tempo de doença (Macuglia, Rieder, \& Almeida, 2012). Especificamente, pacientes com um quadro de DE apresentam dificuldades na tomada de decisões, impulsividade, desatenção, diminuição da motivação, insensibilidade, dificuldades de planejamento, além de traçar metas irrealistas, não avaliar as consequências de seus atos e buscar soluções via tentativa-e-erro (Campos-Sousa et al., 2010).

A DP é apontada como a segunda doença neurodegenerativa mais comum entre idosos acarre- tando incapacidades severas e grande impacto financeiro e social (Hastings, 2009). Entretanto, ainda há uma escassez de estudos investigando a natureza e o grau dos prejuízos executivos específicos de pacientes da população brasileira, conhecimento que é importante para profissionais que trabalham com a avaliação neuropsicológica de pacientes com DP. Em virtude disso, o presente trabalho teve como objetivo investigar as funções executivas em adultos saudáveis e com DP e suas peculiaridades entre homens e mulheres nas diferentes fases da doença. $\mathrm{O}$ estudo também buscou explorar as características distintivas entre pacientes com nível leve e moderado à grave da DP.

\section{MÉTODO}

\section{Participantes}

Os participantes foram 30 adultos controles saudáveis, com idades entre 44 e 75 anos $(M=61,40$; $D P=9,36 ; 70,0 \%$ mulheres) e 40 adultos com diagnóstico prévio de DP, com idades entre 44 e 75 anos ( $M=63,33 ; D P=8,44 ; 57,5 \%$ mulheres). Os pacientes clínicos eram provenientes ou do Ambulatório de Distúrbios do Movimento do Hospital de Clínicas de Porto Alegre-RS (HCPA), ou foram indicados por médicos (neurologistas, neurocirurgiões e médicos que atendem no programa Estratégia de Saúde da Família) do município Cruz Alta - RS. Os controles, por sua vez, eram acompanhantes de pacientes ou voluntários que aceitaram participar da pesquisa.

Foram critérios de exclusão para ambos os grupos a presença de distúrbios neurológicos atuais (além da DP); histórico de abuso de álcool e drogas, avaliado pelo Questionário CAGE, a presença de demência ou declínio cognitivo leve avaliados pelo Mini Exame do Estado Mental (MEEM); e ter se submetido à neurocirurgia. Ainda, para o grupo clínico, foi considerado como critério de exclusão a participação em algum programa de reabilitação neuropsicológica.

Além disto, todas as demais variáveis clínicas e sociodemográficas, com exceção da idade, sexo e escolaridade, não apresentaram interferência nos resultados dos testes executivos. As variáveis avaliadas foram o uso de levodopa e outros medicamentos para o tratamento da DP; participação em um programa de reabilitação; o uso de medicação psiquiátrica; presença de ansiedade e depressão, avaliadas através da Escala Hospitalar de Ansiedade e Depressão (HADS), tempo de doença, idade ao receber o diagnóstico e nível socioeconômico.

Aos que atenderam aos critérios de inclusão e exclusão, foram fornecidas informações a respeito do 
estudo e solicitado que manifestassem sua adesão à pesquisa através de assinatura, após a leitura do Termo de Consentimento Livre e Esclarecido. Os participantes responderam a uma série de instrumentos de avaliação, sempre de forma individual, em encontros com duração em torno de uma hora e meia. $\mathrm{O}$ estudo foi aprovado pelo Comitê de Ética do Hospital de Clínicas de Porto Alegre (CEP-HCPA).

A análise descritiva do grupo de pacientes revelou que $67,5 \%$ (27) encontravam-se em uma fase leve da doença, entre os estágios um e dois e meio da Escala Hoehn \& Yahr. Os demais pacientes, 32,5\% (13) encontravam-se nos estágios moderado a grave, de três a cinco da referida escala.

Ambos os grupos estiveram emparelhados $(p>0,05)$ quanto às variáveis idade, escolaridade e sexo. Dentre os controles saudáveis, 53,3\% relataram possuir quatro a oito anos de escolaridade, sendo $60,0 \%$ casados e $51,7 \%$ pertencentes à classe social B2. Quanto ao grupo dos pacientes com DP, 72,5\% relataram ter de quatro a oito anos de escolaridade, sendo $69,2 \%$ casados e $32,5 \%$ pertencentes à classe social C1. Além disso, dentre os pacientes avaliados, apenas $20 \%$ possuíam algum familiar em primeiro grau com DP e $50 \%$ não participavam de nenhuma forma de reabilitação. Informações descritivas sobre os pacientes avaliados são apresentadas na Tabela 1 .

TABELA 1

Caracterização do Grupo de

Pacientes com Doença de Parkinson

\begin{tabular}{lcccc}
\hline \multicolumn{1}{c}{ Variável } & Mínimo & Máximo & $M$ & $D P$ \\
\hline Idade do diagnóstico & 33 anos & 70 & 53,68 & 9,17 \\
Tempo de doença & 1 ano & 29 & 9,75 & 5,74 \\
UPDRS & 1 & 54 & 21,05 & 12,88 \\
Hoehn \& Yahr & 1 & 5 & 2,34 & 0,75 \\
\hline
\end{tabular}

UPDRS: Escala Unificada de Avaliação da Doença de Parkinson

\section{Instrumentos}

Questionário de Dados Clínicos e Sócio-Demográficos - questionário construído pela pesquisadora para mapear características clínicas e sóciodemográficas, tais como idade, sexo, escolaridade, estado civil, idade de início da doença, tempo da doença, entre outras. Constituído também pelo Critério de Classificação Econômica Brasil (ABEP, 2010).

Questionário CAGE (Ewing \& Rouse, 1970): avalia a síndrome de dependência do álcool. A tradução brasileira do CAGE foi realizada por Masur e Monteiro (1983). É constituído por quatro questões referentes ao anagrama Cut-down, Annoyed, Guilty e
Eye-opener, às quais os testandos devem responder "sim" ou "não".

Avaliação Cognitiva Montreal - MoCA (Nasreddine, Phillips, Bédirian, Charbonneaus, \& Whitehead, 2005), instrumento breve de rastreio para deficiência cognitiva leve. Foi traduzido para a população brasileira por Sarmento, Bertolucci e Wajman (2007). Avalia atenção, concentração, funções executivas, memória, linguagem, habilidades visoconstrutivas, conceituação, cálculo e orientação. O tempo de aplicação é de aproximadamente 10 minutos, e o escore total é de 30 pontos, sendo o escore de 26 ou mais considerado normal.

Mini Exame do Estado Mental - MEEM (Folstein, Folstein, \& McHugh, 1975), instrumento para o rastreio do comprometimento cognitivo. Foi adaptado para a população brasileira por Bertolucci, Brucki, Campacci e Juliani (1994). Avalia orientação temporal e espacial, atenção, cálculo, memória, linguagem e capacidade construtiva visual. Adotou-se o ponto de corte de 24 pontos para pessoas com escolaridade acima de nove anos, e de 17 para aqueles com menor escolaridade (Murden, McRae, Kaner, \& Bucknam, 1991).

Escala de Hoehn e Yahr modificada - HY (Hoehn \& Yahr, 1967), que abrange sete estágios de classificação para avaliar a gravidade da DP, e compreende medidas globais de sinais e sintomas que permitem classificar o indivíduo quanto ao nível de incapacidade. Os estágios um, um e meio, dois e dois e meio indicam incapacidade leve, e os estágios de três, quatro e cinco incapacidade moderada à grave. Participaram da amostra deste estudo os pacientes classificados nos estágios de 1 a 3 .

Behavioural Assessment of the Dysexecutive Syndrome - BADS (Wilson, Alderman, Burgess, Emslie, \& Evans, 1996), uma bateria ecológica de testes que avalia as FE, como controle inibitório, planejamento, prioridades, resolução de problemas, flexibilidade cognitiva e alterações comportamentais. $\mathrm{O}$ instrumento foi traduzido e adaptado para o português brasileiro pela autora principal deste artigo, e encontrase em fase de validação. A BADS é composta por 30 questões divididas entre seis subtestes somando uma pontuação máxima de 24 pontos além de dois questionários. Este escore bruto é transformado em um escore padronizado através da consulta à tabela normativa do instrumento (escore corrigido).

Teste de Stroop - versão Victoria (Stroop, 1935). Trata-se de uma medida de atenção seletiva e de flexibilidade mental. É composto por três cartões, contendo vinte e quatro estímulos cada um. O instrumento foi normatizado para uma amostra brasileira por Duncan (2006). 
WAIS III - Wechsler Adult Intelligence Scale (Wechsler, 1997), considerada um instrumento padrãoouro de avaliação intelectual que permite investigar componentes específicos como FE. Foi adaptada, validada e normatizada para amostras brasileiras por Nascimento (2000). Abrange idades entre 16 e 89 anos e está dividida em quatro Índices Fatoriais, cada um composto por vários subtestes. Para fins deste estudo foram utilizados o Índice Memória de Trabalho e o Índice Velocidade de Processamento e seus respectivos sub-testes: Span de Dígitos, Aritmética, Sequência de Números e Letras (para avaliação da Memória de Trabalho), e Procurar Símbolos (para avaliação da Velocidade de Processamento. O subteste Códigos não foi utilizado em função das dificuldades motoras dos pacientes com Parkinson.

Escala Hospitalar de Ansiedade e Depressão HADS (Zigmond \& Snaith, 1983) - escala autoaplicável, composta por 14 itens de múltipla escolha, dividida em duas subescalas, sendo uma voltada para a avaliação da ansiedade (HADS-A) e a outra para a depressão (HADS-D). Foi validada para a população brasileira por Botega, Bio, Zomignani, Garcia Jr. e Pereira (1995) que encontraram alfa de Cronbach de 0.68 e 0.77 para as subescalas respectivamente

\section{Análise dos Dados}

Para a comparação dos grupos, foi utilizado o Teste Mann-Whitney. As variáveis categóricas foram analisadas com o Teste de Qui-quadrado. Todas as análises foram realizadas utilizando o software Statistical Package for the Social Sciences (SPSS), versão 18.0. Foi adotado o critério de significância $\mathrm{p}<0,05$.

\section{RESULTADOS}

As análises de comparações de postos revelaram um desempenho significativamente inferior para os pacientes parkinsonianos quando comparados aos indivíduos controles nos escores totais nos testes de avaliação neuropsicológica MoCA $(p=0,005)$, MEEM $(p=0,024)$ e BADS $(p=0,001)$, assim como no Teste Stroop - Cartão 1 (número de erros) $(p=0,017)$, e nos subtestes WAIS-III Sequência de Números e Letras $(p=0,019)$ e Procurar Símbolos $(p=0,001)$. Na sequência, buscou-se investigar variáveis influentes no desempenho dos pacientes nos instrumentos aplicados. Dentre o grupo dos pacientes, observou-se uma influência significativa das variáveis escolaridade, sexo e idade em instrumentos de avaliação das FE. Como visto na Tabela 2, indivíduos com maior escolaridade, com idade menor, e do sexo masculino apresentaram desempenho significativamente superior na maioria dos aspectos avaliados.

Com base na escala HY, dividiram-se os pacientes em dois grupos distintos, de acordo com a etapa da doença em que cada um encontrava-se (leve vs. moderada à grave). A análise descritiva revelou que $67,5 \%$ (27) dos pacientes se encontravam em uma fase leve da doença e os demais, 32,5\% (13) encontravamse nos estágios moderado a grave. Então, ambos os grupos de pacientes foram comparados quanto ao seu desempenho executivo. Como esperado, as comparações entre estes dois grupos evidenciaram maior comprometimento cognitivo no grupo de pessoas na fase moderada à grave da doença, embora essa diferença não tenha sido significativa para todos os instrumentos (Tabela 3).

Na sequência, os pacientes parkinsonianos foram classificados, a partir do ponto de corte da BADS, no qual escores $<70$ indicam a presença de $\mathrm{DE}$ (Wilson et al., 1996). Assim, verificou-se que 72,5\% $(n=29)$ dos pacientes com DP possuíam disfunção executiva. Uma comparação subsequente feita entre pacientes com e sem DE é apresentada na Tabela 4, na qual é possível ver diferenças significativas em diversos aspectos cognitivos. Ainda foi observada uma associação entre a fase de desenvolvimento da DP com a faixa etária em que se encontravam os pacientes. Entre os pacientes na fase leve da doença $55,6 \%(\mathrm{n}=15)$ possuíam entre 40 e 59 anos de idade, já entre o grupo com doença moderada à grave $100 \%$ $(\mathrm{n}=13)$ tinham de 60 a 75 anos de idade. Além disto, houve um acréscimo no número de pacientes com disfunção executiva entre as fases (de 64,6 \% para $66,7 \%$ ), distúrbio já prevalente em ambas as etapas da DP.

Além disso, o teste Qui-quadrado foi empregado para investigar diferenças entre ambos os grupos de pacientes quanto ao sexo e às categorias de escolaridade. O grupo com disfunção executiva apresentou escolaridade significativamente inferior ao grupo sem disfunção executiva (Fisher's exact test, $p=0,042$ ). Mas especificamente, a maioria dos pacientes com disfunção executiva $(82,8 \%, n=24)$ possuía quatro a oito anos de estudo. Em contraste, $54,5 \%$ dos pacientes sem disfunção executiva possuíam nove ou mais anos de escolaridade. Em relação ao sexo, houve uma frequência $(72,7 \%, n=8)$ significativamente mais alta de indivíduos do sexo masculino no grupo de pacientes sem disfunção executiva (Fisher's exact test, $p=0,030$ ). Uma investigação mais aprofundada, explorando outros aspectos sociodemográficos não revelou outras diferenças significativas entre os grupos. 
TABELA 2

Diferenças nos Escores dos Testes Cognitivos entre Pacientes com DP.

\begin{tabular}{|c|c|c|c|c|c|}
\hline \multirow{2}{*}{ Escolaridade } & \multicolumn{2}{|c|}{4 a 8 anos } & \multicolumn{2}{|c|}{9 ou mais } & \multirow{2}{*}{$p^{*}$} \\
\hline & $M$ & $D P$ & $M$ & $D P$ & \\
\hline BADS total & 9,14 & 3,31 & 11,82 & 3,68 & 0,028 \\
\hline BADS escore corrigido & 58,62 & 16,93 & 72,09 & 16,16 & 0,030 \\
\hline Aritmética & 7,90 & 2,19 & 11,00 & 3,53 & 0,021 \\
\hline Procurar símbolos & 10,48 & 5,45 & 18,64 & 8,00 & 0,003 \\
\hline Stroop 1 tempo & 29,00 & 16,42 & 20,18 & 9,17 & 0,002 \\
\hline Stroop 2 tempo & 45,90 & 30,29 & 30,27 & 18,19 & 0,038 \\
\hline MoCA abstração & 0,62 & 0,68 & 1,45 & 0,82 & 0,007 \\
\hline MEEM total & 23,41 & 3,43 & 26,09 & 3,05 & 0,033 \\
\hline MEEM atenção e cálculo & 1,66 & 1,78 & 3,64 & 1,75 & 0,005 \\
\hline \multirow{2}{*}{ Idade } & \multicolumn{2}{|c|}{40 a 59 anos } & \multicolumn{2}{|c|}{60 a 75 anos } & \multirow{2}{*}{$p^{*}$} \\
\hline & $M$ & $D P$ & $M$ & $D P$ & \\
\hline Dígitos total & 10,80 & 2,27 & 9,33 & 2,32 & 0,035 \\
\hline Stroop 2 tempo & 37,93 & 32,83 & 40,29 & 18,83 & 0,050 \\
\hline Stroop 3 tempo & 45,27 & 27,00 & 57,67 & 24,86 & 0,028 \\
\hline Stroop 3 erros & 1,00 & 2,00 & 2,92 & 3,12 & 0,010 \\
\hline Hoehn \& Yahr & 2,03 & 0,35 & 2,41 & 0,70 & 0,022 \\
\hline \multirow{2}{*}{ Sexo } & \multicolumn{2}{|c|}{ Masculino } & \multicolumn{2}{|c|}{ Feminino } & \multirow{2}{*}{$p^{*}$} \\
\hline & $M$ & $D P$ & $M$ & $D P$ & \\
\hline Aritmética & 9,76 & 2,73 & 7,91 & 2,67 & 0,016 \\
\hline Stroop 3 tempo & 45,65 & 29,92 & 58,50 & 21,76 & 0,015 \\
\hline BADS total & 11,82 & 3,28 & 8,73 & 2,86 & 0,003 \\
\hline BADS escore corrigido & 70,35 & 15,50 & 58,14 & 15,11 & 0,012 \\
\hline Cartas & 2,12 & 1,49 & 1,14 & 0,99 & 0,027 \\
\hline Programa ação & 3,18 & 1,13 & 2,59 & 1,22 & 0,042 \\
\hline MEEM orientação & 9,76 & 0,44 & 9,09 & 1,02 & 0,019 \\
\hline
\end{tabular}

* Teste de Mann-Whitney.

BADS: Behavioural Assessment of Dysexecutive Syndrome; MEEM: Mini Exame do Estado Mental; MoCA: Avaliação Cognitiva Montreal.

TABELA 3

Comparações dos Desempenhos em Instrumentos Cognitivos e Idade entre os Pacientes com DP Leve vs. Moderada à Grave

\begin{tabular}{|c|c|c|c|c|c|}
\hline & \multicolumn{2}{|c|}{ Leve $(N=27)$} & \multicolumn{2}{|c|}{ Moderada à grave $(N=13)$} & \multirow{2}{*}{$p^{*}$} \\
\hline & $\mathrm{M}$ & $\mathrm{DP}$ & $\mathrm{M}$ & $\mathrm{DP}$ & \\
\hline \multicolumn{6}{|l|}{ WAIS - III } \\
\hline Aritmética & 9,43 & 3,16 & 7,42 & 1,93 & 0,027 \\
\hline Procurar Símbolos & 14,52 & 7,10 & 10,00 & 6,93 & 0,019 \\
\hline \multicolumn{6}{|l|}{ Stroop } \\
\hline Stroop 1 tempo & 26,87 & 15,45 & 32,67 & 10,09 & 0,034 \\
\hline Stroop 2 tempo & 33,83 & 24,30 & 48,17 & 19,16 & 0,002 \\
\hline Stroop 3 tempo & 43,48 & 18,71 & 69,75 & 29,30 & 0,005 \\
\hline \multicolumn{6}{|l|}{ MEEM } \\
\hline Total & 25,30 & 2,96 & 22,25 & 3,47 & 0,004 \\
\hline \multicolumn{6}{|l|}{$\mathrm{MoCa}$} \\
\hline Visuo execução & 3,26 & 1,45 & 2,25 & 1,71 & 0,056 \\
\hline Orientação & 5,91 & 0,29 & 5,42 & 0,67 & 0,023 \\
\hline Total & 20,22 & 5,23 & 14,67 & 4,60 & 0,002 \\
\hline \multicolumn{6}{|l|}{ BADS } \\
\hline Programa ação & 3.09 & 1,08 & 2,25 & 1,42 & 0,023 \\
\hline Total & 11,17 & 3,46 & 8,08 & 2,19 & 0,004 \\
\hline DEX independente cognitivo & 4,61 & 2,78 & 8,50 & 5,62 & 0,055 \\
\hline DEX independente total & 18,48 & 11,77 & 35,83 & 20,06 & 0,018 \\
\hline
\end{tabular}

* Teste Mann-Whitney 
TABELA 4

Comparação dos Escores nos Testes Neuropsicológicos entre Pacientes com e sem Disfunção Executiva.

\begin{tabular}{|c|c|c|c|c|c|}
\hline & \multicolumn{2}{|c|}{$\operatorname{Sem} D E^{*}(N=11)$} & \multicolumn{2}{|c|}{ Com $D E^{*}(N=29)$} & \multirow{2}{*}{$p^{* *}$} \\
\hline & $M$ & $D P$ & $M$ & $D P$ & \\
\hline \multicolumn{6}{|l|}{ WAIS - III } \\
\hline Aritmética & 9,70 & 3,38 & 8,63 & 2,84 & $<0,001$ \\
\hline Sequencia Números Letras & 6,03 & 2,88 & 4,45 & 2,43 & $<0,001$ \\
\hline Procurar Símbolos & 18,63 & 7,29 & 12,73 & 7,17 & $<0,001$ \\
\hline \multicolumn{6}{|l|}{ Stroop } \\
\hline Stroop 1 tempo & 25,50 & 9,91 & 29,83 & 15,85 & 0,017 \\
\hline Stroop 2 tempo & 31,57 & 14,17 & 41,60 & 28,17 & 0,003 \\
\hline Stroop 3 tempo & 49,30 & 24,54 & 52,90 & 26,08 & 0,003 \\
\hline Stroop 3 erros & 1,57 & 3,14 & 2,18 & 2,87 & 0,043 \\
\hline \multicolumn{6}{|l|}{ MEEM } \\
\hline Atenção.Cálculo & 2,40 & 2,18 & 2,20 & 1,96 & 0,002 \\
\hline Linguagem & 7,70 & 0,65 & 7,48 & 0,82 & 0,018 \\
\hline Total & 26,10 & 2,56 & 24,15 & 3,51 & 0,002 \\
\hline \multicolumn{6}{|l|}{$\mathrm{MoCa}$} \\
\hline Visuo execução & 3,77 & 1,25 & 2,83 & 1,63 & 0,004 \\
\hline Atenção & 4,27 & 1,72 & 3,48 & 1,77 & 0,001 \\
\hline Linguagem & 1,47 & 1,01 & 1,73 & 1,01 & 0,015 \\
\hline Abstração & 1,10 & 0,85 & 0,85 & 0,80 & 0,044 \\
\hline Total & 21,50 & 4,28 & 17,98 & 5,82 & 0,001 \\
\hline \multicolumn{6}{|l|}{ BADS } \\
\hline Cartas & 2,30 & 1,21 & 1,53 & 1,32 & $<0,001$ \\
\hline Mapa Zôo & 0,87 & 0,94 & 0,43 & 0,81 & 0,007 \\
\hline Seis Elementos & 2,60 & 1,19 & 2,25 & 1,15 & 0,001 \\
\hline Escore corrigido & 83,89 & 10,37 & 57,00 & 10,67 & $<0,001$ \\
\hline Escore total & 14,44 & 2,45 & 8,62 & 2,15 & $<0,001$ \\
\hline
\end{tabular}

* Os pacientes foram classificados nas categorias com e sem DE a partir do ponto de corte $<70$ da BADS.

** Teste Mann-Whitney.

\section{DISCUSSÃO}

A amostra de pacientes se caracterizou em sua maioria por idosos, perfil característico dos portadores de DP. A literatura indica que tanto no envelhecimento normal como no patológico, as FE tendem a estar prejudicadas (Zibetti et al., 2010). Uma possível explicação para esse declínio é o desgaste neurobiológico natural dos lobos frontais. Duas teorias tentam explicar este declínio. Uma delas refere-se à ontogênese; ou seja, os lobos frontais, por serem as áreas que mais tardiamente se desenvolveram no ser humano, são também as mais suscetíveis ao processo de envelhecimento (Parente \& Wagner, 2009). A outra teoria baseia-se nas alterações estruturais dessas áreas, em que se verifica uma redução neuronal frontal de 10 a $17 \%$, maior do que a ocorrida nos córtices temporal, parietal e occipital (Porto, Carvalho, Neves, Novo, \& Castelli, 2010).

As alterações executivas ocorrem de modo gradual em idosos saudáveis até os 60 anos, acelerando-se a partir dos 70 anos (Papalia \& Feldman, 2013). Este dado justifica a forma como os participantes deste estudo foram divididos, de acordo com sua faixa etária (de 40 a 59 anos e de 60 a 75 anos). Foram notadas diferenças significativas entre esses grupos para a perseveração, flexibilidade mental, atenção, concentração, memória de trabalho, velocidade de processamento, inibição dos impulsos e percepção visual. Percebe-se que não há uma homogeneidade na forma como a idade afeta os diversos componentes cognitivos. Pesquisadores não constataram um padrão uniforme de mudanças relacionadas com a idade para todas as habilidades intelectuais. Os dados indicam que o desenvolvimento 
cognitivo é irregular na idade adulta, ou seja, é marcado por ganhos e perdas em épocas e habilidades diferentes (Papalia \& Feldman, 2013).

Em relação ao sexo, destaca-se na amostra estudada, o melhor desempenho dos homens em tarefas executivas de atenção seletiva e concentrada, flexibilidade cognitiva, perseveração, planejamento, resolução de problemas e inibição de impulsos, indicando diferenças no modo como os sexos são afetados pela doença. Apesar da questão de gênero ser um dado relevante para a escolha de testes adequados para a avaliação destes pacientes, assim como para a organização de programas de reabilitação específicos, abordagens sobre as diferenças cognitivas entre homens e mulheres com DP têm sido pouco exploradas (Miller \& Cronin-Golomb, 2010). Em um estudo sobre o tema Riedel et al. (2008), através de uma avaliação de 873 pacientes com DP, observaram a influência do gênero em suas performances em testes executivos, tendo os homens apresentando melhores desempenhos. Banhato e Nascimento (2007) compararam idosos saudáveis de ambos os sexos em relação às $\mathrm{FE}$ encontrando resultados semelhantes.

Além da idade e do sexo, a escolaridade vem sendo apontada por muitos pesquisadores pelo seu alto impacto sobre a cognição, especialmente determinante no desempenho em tarefas que avaliam FE (Parente, Scherer, Zimmermann \& Fonseca, 2009). Estes autores ressaltam que as diferenças cognitivas entre os grupos etários costumam ser menores do que as identificadas entre os grupos com diferentes níveis de escolaridade, dado encontrado neste estudo. No entanto, somente a alfabetização não responde pelos efeitos sobre a cognição, devendo ser incluído nesta avaliação o tempo de estudo associado às variáveis socioculturais. Merguro et al. (2001) encontraram correlações entre atrofia do lobo frontal e idade em indivíduos com baixa escolaridade. Os autores afirmam que o tempo de escolaridade possui consequências sobre a estrutura cerebral, aumentado o número de sinapses e a vascularização cerebral. No presente estudo, a amostra de pacientes caracterizou-se por apresentar menor escolaridade nos estágios mais avançados da DP.

Pesquisas recentes têm apontado a importância da reserva cognitiva como fator protetor nas mais diversas patologias neurológicas, uma vez demonstrada sua alta correlação com as FE (Siedlecki et al., 2009). Um estudo utilizando ressonância magnética funcional (fMRI) demonstrou que a reserva cognitiva em adultos, potencializada pela escolarização, está relacionada ao tamanho cerebral e à menor utilização de redes neurais, porém mais eficientes, durante a realização de tarefas neuropsicológicas, ao passo que em idosos com pouca reserva cognitiva, ocorre o padrão oposto (Solé-Padullés et al., 2009). Em relação à escolaridade, a amostra estudada foi dividida em dois grupos: de quatro a oito anos e nove ou mais anos de estudo. Esta divisão está de acordo com grande parte dos estudos realizados na América Latina, que utilizam em média grupos de um a quatro anos, cinco a oito e nove ou mais anos de escolaridade (Parente et al., 2009). Em ambos os grupos, a escolaridade apresentou relação com quase todos os testes utilizados na pesquisa, com exceção daqueles que avaliaram organização do tempo, julgamento, pensamento abstrato, nomeação, evocação, orientação, formulação e execução de um plano. Os testes mais sensíveis para diferenciar os grupos clínico e controle foram MoCA (total, viso-executiva e evocação tardia), MEEM (total, evocação e praxia), BADS (total, cartas, procurar chaves e julgamento temporal), Stroop (cartão 1 - erros) e WAIS - III (sequência de números e letras e procurar símbolos). O fraco desempenho dos pacientes para as tarefas de evocação pode ser explicado pela relação entre disfunções executivas e o envelhecimento dos sistemas de memória. O principal modelo que defende essa teoria determina que o envelhecimento da memória possa ser entendido pelo comprometimento das funções frontais, especialmente pelo decréscimo nos processos atencionais (Clemente, \& Ribeiro-Filho, 2008). Importante também considerar a presença de intrusões no processo de memória, seja na evocação ou no reconhecimento, indício de falhas na organização executiva, possivelmente decorrente da pouca organização mental para encontrar as informações retidas e não pela perda de dados por falhas de codificação (Osternack-Pinto, 2005). A menor acurácia atencional, além de relacionar-se com as dificuldades na evocação, também está associada a um pobre desempenho em tarefas de avaliação de percepção visual e velocidade de processamento, como é o caso do teste Procurar símbolos (Zibetti et al., 2010).

Alguns autores sugerem que alterações das funções executivas estão na base do comprometimento das habilidades viso-espaciais, assim como da memória e linguagem (Caballol, Martí, \& Tolosa, 2007). Disfunções visuoespaciais derivam da dificuldade em realizar análise contextual e inibir a resposta mais imediata solicitada pelo estímulo (atenção seletiva). Esta função avaliada pela escala MoCA, através dos subtestes das trilhas, desenho do relógio e cópia de uma figura, pode ser explicada pelo comprometimento frontal, comum em pacientes com DP (Piovezan, Teive, Piovesan, Mader, \& Werneck, 2007). Nesse sentido, deve-se considerar que esses testes envolveram habilidade motora, prejudicada nestes pacientes. 
Os resultados do teste Stroop, nos três cartões, revelaram uma pequena lentidão nas respostas dos pacientes, embora não significativa para diferenciar os grupos. Resultado semelhante foi encontrado no estudo de Osternack-Pinto (2005). Como essa tarefa envolve rapidez sem envolvimento motor, pode-se dizer que o maior número de erros ocorridos entre pacientes no cartão um se deve às dificuldades na atenção focal, mas não à inibição dos impulsos. Pacientes com DP produzem baixos escores em tarefas de controle atencional que exigem bom funcionamento da memória de trabalho. No presente estudo, isso ficou evidente nos resultados encontrados nos teste Sequência de números e letras (SNL), mas não no teste Dígitos ordem inversa. Os dois instrumentos apresentaram correlação moderada positiva $(r=0,56$; $p=<0,001)$, indicando que ambos medem a mesma função.

Os resultados obtidos com a BADS evidenciaram maior comprometimento executivo dos sujeitos do grupo clínico do que do controle. Além disto, o instrumento correlacionou-se com todos os instrumentos utilizados nesta pesquisa, com exceção do Dígitos ordem direta, Stroop (cartão 1 - erros) e MEEM (evocação). Apenas dois subtestes da BADS não se mostraram úteis para a diferenciação entre os grupos experimentais: Programa ação (que avalia planejamento e resolução de problemas) e Seis elementos modificado (que avalia planejamento, organização e monitoramento do comportamento). No estudo de Perfetti et al. (2010), o subteste Programa ação também não apresentou poder discriminativo entre os grupos. Uma possível explicação para este dado é que o escore do teste não leva o tempo de execução da tarefa em consideração, permitindo a pessoas que, embora apresentem alterações do funcionamento executivo, consigam executar a tarefa.

A escala Hoehn \& Yahr correlacionou-se com grande parte dos testes de avaliação cognitiva, especialmente as tarefas executivas. A partir dos escores desta escala foi possível encontrar duas classes de pacientes com DP (leve e de moderada à grave). A DE estava presente nos dois grupos, reforçando os achados da literatura que diz que este transtorno está presente desde o início da DP (Mckinlay et al., 2010).

Escolaridade e sexo apresentaram associação significativa com a variável disfunção executiva. A escolaridade, como discutido acima, é apontada como influente em diversos processos neuropsicológicos (Parente et al., 2009), e mostrou-se um fator protetor para alterações executivas em pacientes com DP neste estudo. O sexo, como relata a literatura, também se mostrou uma variável significativamente influente no grau das alterações executivas, indicando maiores prejuízos entre as mulheres, dado crucial para a abordagem destes pacientes.

O estudo apresenta algumas limitações como uma amostra pequena, o não controle de variáveis como a presença de comorbidades clínicas que podem ter influência sobre o desempenho das FE como diabetes e hipertensão. Ainda seria ideal o ajuste do ponto de corte da BADS de acordo com a escolaridade, mas esta informação não é contemplada no manual original do instrumento e ainda não existem normas brasileiras para o mesmo. Desta forma, sugere-se futuros estudos que contemplem estas variáveis para o aprofundamento do conhecimento sobre os distúrbios cognitivos presentes em pacientes com DP.

\section{REFERÊNCIAS}

Banhato, E. F. C. \& Nascimento, E. do. (2007). Função executiva em idosos: um estudo utilizando subtestes da escala WAIS-III. Psico-USF, 12(1), 65-73.

Bertolucci, P. H. F., Brucki, S. M. D., Campacci, S. R., \& Juliani, Y. (1994). O mini-exame do estado mental em uma população geral: impacto da escolaridade. Arquivos de Neuropsiquiatria, 52, 1-7. http://dx.doi.org/10.1590/S0004282X1994000100001

Botega, N. J., Bio, M. R., Zomignani, M. A., Garcia Jr., C., \& Pereira, W. A. B. (1995). Transtornos de humor em enfermaria de clínica médica e validação da escala de medida (HADS) de ansiedade e depressão. Revista de Saúde Pública, 29(5), 359-363. http://dx.doi.org/10.1590/S0034-89101995000500004

Caballol, N., Martí, M. J., \& Tolosa, E. (2007). Cognitive dysfunction and dementia in Parkinson's disease. Movement Disorders, 22(17), 358-366. http://dx.doi.org/10.1002/mds.21677

Campos-Sousa, I. S., Campos-Sousa, R. N., Ataíde Jr., L., Soares, M. M. de B., \& Almeida, K. J. (2010). Executive dysfunction and motor symptoms in Parkinson's disease. Arquivos de Neuropsiquiatria, 68(2), 246-251. http://dx.doi. org/10.1590/S0004-282X2010000200018

Chan, R. C. K., Shun, D., Toulopoulou, T., \& Chen, E. V. H. (2008). Assessment of executive functions: review of instruments and identification of critical issues. Archives of Clinical Neuropsychology, 23, 201-216. http://dx.doi. org/10.1016/j.acn.2007.08.010 
Clemente, R. S. G. \& Ribeiro-Filho, S. T. (2008). Comprometimento cognitivo leve: aspectos conceituais, abordagem clínica e diagnóstica. Revista do Hospital Universitário Pedro Ernesto, 7(1), 68-77.

Duncan, M. T. (2006). Obtenção de dados normativos para desempenho no teste de Stroop num grupo de estudantes do ensino fundamental em Niterói. Jornal Brasileiro de Psiquiatria, 55(1), 42-48.

Ewing, J. A. \& Rouse, B. A. (1970). Identifying the hidden alcoholic. In Program and abstracts of the 29th internacional congress on alcohol and drug dependence. Sidney. Australia.

Folstein, M. F., Folstein, S. E., \& McHugh, P. R. (1975). Mini-mental state; a pratical method for grading the cognitive state of patients for clinician. Journal of Psychiatric Research, 12, 189-198. http://dx.doi.org/10.1016/0022-3956 (75)90026-6

Galhardo, M. M. de A. M. C., Amaral, A. K. de F. J. do, \& Vieira, A. C. de C. (2009). Caracterização dos distúrbios cognitivos na doença de Parkinson. Revista CEFAC, 11(2), 251-257. http://dx.doi.org/10.1590/S1516-18462009000600015

Hamdan, A. C. \& Pereira, A. P. de A. (2009). Avaliação neuropsicológica das funções executivas: considerações metodológicas. Psicologia Reflexão e Crítica, 22(3), 386-393. http://dx.doi.org/10.1590/S0102-79722009000300009

Hoehn, M. M. \& Yahr, M. D. (1967). Parkinsonism: onset progression and mortality. Neurology, 17(5), 427-442. http:// dx.doi.org/10.1212/WNL.17.5.427

Hastings, T. G. (2009). The role of dopamine oxidation in mitochondrial dysfunction: implications for Parkinson's disease. Journal Bioenergetics and Biomembranes, 41(6), 469-472. http://dx.doi.org/10.1007/s10863-009-9257-z

Kamei, S., Morita, A., Serizawa, K., Mizutani, T., \& Hirayanagi, K. (2010). Journal of Clinical Neurophysiology, 27(3), 193-197. http://dx.doi.org/10.1097/WNP.0b013e3181dd4fdb

Macuglia, G. R., Rieder, C. R. M., \& Almeida, R. M. M. (2012). Funções executivas na doença de Parkinson: revisão da literatura. Revista Psico, 43(4), 552-561.

Malloy-Diniz, L. F., Paula, J. J. de, Loschiavo-Alvares, F. Q., Fuentes, D., \& Leite, W. B. (2010). Exame das funções executivas. In L. F. Malloy-Diniz, D. Fuentes, \& P. Mattos. Avaliação neuropsicológica. Porto Alegre: Artmed.

Masur, J. \& Monteiro, M. G. (1983). Validation of the "CAGE" alcoholism screening test in a Brazilian psychiatric impatient hospital setting. Brazilian Journal of Medical and Biological Research, 16, 215-218.

Mckinlay, A., Grace, R. C., Dalrymple-Alford, J. A., \& Roger, D. (2010). Characteristics of executive function impairment in Parkinson's disease patients without dementia. Journal of the International Neuropsychological Society, 16 , 268-277. http://dx.doi.org/10.1017/S1355617709991299

Meguro, K., Shimada, M., Yamaguchi, S., Ishizaki, J., Ishii, H., Shimada, Y., Sato, M., Yamadori, A., \& Sekita, Y. (2001). Cognitive function and frontal lobe atrophy in normal elderly adults: Implications for dementia not as agingrelated disorders and the reserve hypothesis. Psychiatry and Clinical Neurosciences, 55(6), 565-72. http://dx.doi. org/10.1046/j.1440-1819.2001.00907.x

Miller, I. N. \& Cronin-Golomb, A. (2010). Gender differences in Parkinson's disease: Clinical characteristics and cognition. Movement Disorders, 25(16), 2695-2703. http://dx.doi.org/10.1002/mds.23388

Murden, R. A., McRae, T. D., Kaner, S., \& Bucknam, M. E. (1991). Mini-Mental State Exam scores vary with education in blacks and whites. Journal of the American Geriatrics Society, 39, 149-155.

Nascimento, E. (2000). Adaptação e validação do teste WAIS-III para um contexto brasileiro. Tese de doutorado, Programa de Pós-Graduação em Psicologia, Universidade de Brasília, Brasília, Distrito Federal.

Nasreddine, Z. S., Phillips, N. A., Bédirian, V., Charbonneau, S., Whitehead, V., Collin, I., Cummings, J. L., \& Chertkow, H. (2005). The Montreal cognitive assessment, MoCA: a brief screening tool for mild cognitive impairment. Journal of the American Geriatrics Society, 53, 695-699. http://dx.doi.org/10.1111/j.1532-5415.2005.53221.x

Osternack-Pinto, K. (2005). Análise comparativa das funções neuropsicológicas de portadores de doença de Parkinson em estágios inicial e avançado: uma determinação de padrões para diagnóstico em população brasileira. Tese de doutorado, Programa de Pós-Graduação em Medicina, Universidade de São Paulo, São Paulo.

Papalia, D. E. \& Feldman, R. D. (2013). Desenvolvimento Humano (12ª ed.). Porto Alegre: Artmed.

Parente, M. A. de M. P., \& Wagner, G. P. (2009). Teorias abrangentes sobre envelhecimento cognitivo (pp. 30-45). In M. A. de M. P. Parente e cols. Cognição e envelhecimento. Porto Alegre: Artmed.

Parente, M. A. de M. P., Scherer, L. C., Zimmermann, N., \& Fonseca, R. P. (2009). Evidências do papel da escolaridade na organização cerebral. Revista Neuropsicologia Latinoamericana, 1(1), 72-80.

Perfetti, B., Varanese, S., Mercuri, P., Mancino, E., Saggino, A., \& Onofrj, M. (2010). Behavioural assessment of dysexecutive syndrome in Parkinson's disease without dementia: a comparison with other clinical executive tasks. Parkinsonism \& Related Disorders, 16(1), 46-50. http://dx.doi.org/10.1016/j.parkreldis.2009.07.011

Piovezan, M. R., Teive, H. A. G., Piovesan, El J., Mader, M. J., \& Werneck, L. C. (2007). Cognitive function assessment in idiopathic Parkinson's disease. Arquivos de Neuropsiquiatria, 65(4-A), 942-946. http://dx.doi.org/10.1590/S0004282X2007000600003

Porto, I., Carvalho, F. A. H. de, Neves, V. T. das, Novo, M. S., \& Castelli, C. M. (2010). Correlação entre níveis de autoestima, performance cognitiva e de memória em idosos: uma visão ecológica. Revista Ambiente e Educação, 15(1), 187-206. 
Riedel, O., Klotsche, J., Spottke, A., Deuschl, G., Förstl, H., Henn, F., Heuser, I., Oertel, W., Reichmann, H., Riederer, P., Trenkwalder, C., Dodel, R., \& Wittchen, H. U. (2008). Cognitive impairment in 873 patients with idiopathic Parkinson's disease. Results from the german study on epidemiology of Parkinson's disease with dementia (GEPAD). Journal of Neurology, 255(2), 255-264. http://dx.doi.org/10.1007/s00415-008-0720-2

Sarmento, A. R. L., Bertolucci, P. H. F., \& Wajman, J. R. (2007). Montreal cognitive assessment: versão experimental brasileira. Recuperado em 13 set. 2010, disponível em: http://www.mocatest.org

Siedlecki, K. L., Stern, Y., Reuben, A., Sacco, R. L., Elkind, M. S. V., \& Wright, C. B. (2009). Construct validity of cognitive reserve in a multiethnic cohort: The Northern Manhattan Study. Journal of the International Neuropsychological Society, 15, 558-569. http://dx.doi.org/10.1017/S1355617709090857

Solé-Padullés, C., Bartrés-Faz, D., Junqué, C., Vendrell, P., Rami, L., Clemente, I. C., Bosch, B., Villar, A., Bargalló, N., Jurado, M. A., Barrios, M., \& Molinuevo, J. L. (2009). Brain structure and function related to cognitive reserve variables in normal aging, mild cognitive impairment and Alzheimer's disease. Neurobiology of Aging, 30, 1114-1124. http://dx.doi.org/10.1016/j.neurobiolaging.2007.10.008

Stroop, J. R. (1935). Studies of interference in serial verbal reactions. Journal of Experimental Psychology, 18 , 643-662. http://dx.doi.org/10.1037/h0054651

Wechsler, D. (1997). Wechsler adult intelligence scale (3rd ed.). San Antonio: Psychological Corporation.

Wilson, B. A., Alderman, N., Burgess, P. W., Emslie, H., \& Evans, J. J. (1996). The behavioural assessment of the dysexecutive syndrome. Bury St Edmunds: Thames Valley Company.

Zibetti, M. R., Gindri, G., Pawlowski, J., Fumagalli, J. F. de S., Parente, M. A. De M. P., Bandeira, D. R., Fachel, J. M. G., \& Fonseca, R. P. (2010). Estudo comparativo de funções neuropsicológicas entre grupos etários de 21 a 90 anos. Revista Neuropsicologia Latinoamericana, 2(1), 55-67.

Zigmond, A. S., \& Snaith, R. P. (1983). The hospital anxiety and depression scale. Acta Psychiatrica Scandinavica, 67, 361-370. http://dx.doi.org/10.1111/j.1600-0447.1983.tb09716.x

\footnotetext{
Autores:

Endereço para correspondência:

Rosa Maria Martins de Almeida

Universidade Federal do Rio Grande do $\mathrm{Su}$

Rua Ramiro Barcelos, 2600 - Bairro Santa Cecília

CEP 90035-003 Porto Alegre, RS, Brasil

E-mail: rosa_almeida@yahoo.com rosa.almeida@ufrgs.br
}

GREICI RÖSSLER MACUGLIA - Doutoranda, Universidade Federal do Rio Grande do Sul.

Carlos Roberto de Mello Rieder - Doutor, Universidade Federal do rio Grande do Sul.

LAIS BRoch TRENTINI - Graduanda, Universidade Federal do Rio Grande do Sul.

Nelson Hauck FilHo - Doutor, Programa de Pós-Graduação Stricto Sensu em Psicologia, Universidade São Francisco, Itatiba, SP, Brasil.

ANDRÉ Luiz Moraes - Mestre, Universidade Federal do Rio Grande do Sul.

Rosa Maria Martins de Almeida - Pós Doutorado, Universidade Federal do Rio Grande do Sul.

Recebido em: 30.09 .13

Aceito em: 12.05 .14 